\title{
Impacto de la influencia de la familia en el negocio, sobre el proceso de gestión financiera y la generación del valor en el sector textil- confección en la Ciudad de Medellín y su Área Metropolitana, Colombia
}

Impact of the influence family in businesses on the financial management process and value generation in the textile-clothing sector in Medellin and its Metropolitan Area, Colombia

\section{Paula Andrea Molina Parra ${ }^{1 *}$, Iván Alonso Montoya Restrepo², Sergio Botero Botero ${ }^{2}$}

${ }^{1}$ Politécnico Colombiano Jaime Isaza Cadavid, Colombia

${ }^{2}$ Universidad Nacional de Colombia, Colombia

Recibido el 20 de junio de 2018; aceptado el 13 de agosto de 2018

Disponible en Internet el: 20 de agosto de 2019

\section{Resumen}

El propósito de esta investigación es medir el impacto de la Influencia de la familia sobre el proceso de gestión financiera y la generación de valor en las empresas del sector textil-confección en la Ciudad de Medellín y su área metropolitana. La primera relación se midió a través de un método avanzado de estadística multivariada, la correlación canónica y la segunda con una regresión múltiple, en una muestra probabilística de 100 empresas. Se encontró que la influencia de la familia impacta positivamente la planeación financiera, el control financiero, las decisiones de inversión, la gestión del capital de trabajo y las decisiones de financiamiento; el impacto causado sobre el EVA es positivo pero débil.

\footnotetext{
*Autor para correspondencia

Correo electrónico pamolina@elpoli.edu.co (P.A. Molina Parra).

La revisión por pares es responsabilidad de la Universidad Nacional Autónoma de México. 
Código JEL: M10, L21, G32

Palabras clave: Empresas familiares; Gestión financiera; Generación de valor; Influencia de la familia

\begin{abstract}
The purpose of this research was to measure the impact of the family influence on the financial management process and value generation in the textile-clothing sector in the Medellin metropolitan area. The former was measured with an advanced multivariate statistical method, canonical correlation, and the latter was measured with multiple regression, in a probabilistic sample of 100 companies. It was found that family-ownership had a positive impact on financial planning, financial control, investment decisions, working capital management and financing decisions. The impact on EVA was also positive, but weak.
\end{abstract}

JEL codes: M10, L21, G32

Keywords: Family businesses; Financial management; Value generation; Family influence

\title{
Introducción
}

Las empresas de familia son definidas como "Aquellas organizaciones donde una familia posee como mínimo el $51 \%$ de la propiedad, y algunos de sus miembros hagan parte de la gestión, con la participación en cargos directivos y/o ejecutivos, y del control de la empresa, mediante su participación en órganos de gobierno y en los procesos de la toma de decisiones, con la visión de darle continuidad al negocio" (Molina, 2017, p. 31). Estas organizaciones han demostrado con cifras que tienen mayores dificultades para crecer y permanecer en el tiempo, porque no se planea el proceso de sucesión de la propiedad y la gestión financiera, además se desconoce las diferentes formas en que las familias pueden influir en las finanzas corporativas y en las decisiones de gobernabilidad (González, Guzmán, Pombo y Trujillo, 2014). Dado que un número significativo de empresas a nivel internacional son familiares, se hace necesario reproducir esfuerzos para conocer cómo sus elementos diferenciadores frente a las empresas no familiares contribuyen a un mejor o peor desempeño. La falta de homogeneidad en los resultados de estudios anteriores sugiere que las relaciones entre la empresa familiar y el rendimiento de las empresas son complejas y muy probablemente moderadas o intervenidas por factores que no han sido incluidos en estos análisis.

Los resultados que se obtuvieron de la revisión de los estudios que se han hecho a nivel internacional, permiten concluir que no es posible establecer una relación directa entre la influencia de la familia en el negocio y la rentabilidad de la empresa.

La evidencia empírica es contradictoria, no existe acuerdo entre los académicos: existen trabajos que concluyen que las empresas familiares tienen un mejor desempeño que las 
empresas no familiares; por ejemplo: en España (Santana y Cabrera, 2001); en América (Villalonga y Amit, 2006; Anderson y Reeb, 2003) hallan que las empresas familiares tienen un mejor desempeño que las no familiares y que esto se reflejará en el valor futuro de la empresa, especialmente cuando los fundadores trabajan como gerentes. Otros que concluyen que las empresas familiares tienen un peor desempeño o son menos eficientes que las empresas no familiares, debido a la concentración de la propiedad que reduce la diversificación de riesgo financiero y aumenta el costo de capital (Molina, Botero y Montoya, 2017). Estas ineficiencias también son atribuidas a los diferentes conflictos que se pueden presentar entre los diferentes miembros de la familia (Schulze, et al, 2003). Y, por último, se encuentran los estudios que concluyen que no existe diferencia entre el desempeño financiero de ambos tipos de empresas (Molina, Botero y Montoya, 2017).

Sin embargo, es importante mencionar que los anteriores estudios se han realizado en diferentes contextos geográficos y culturales, además que han tenido en cuenta diferentes parámetros para definir las empresas familiares, los cuales pueden ser determinantes en los resultados encontrados.

En algunos de los estudios revisados se muestra que los familiares propietarios influyen las decisiones financieras y estratégicas en estos negocios, lo que impacta el accionar de las empresas, su valoración y su estructura financiera (Poutziouris, 2001; Romano, Tanewski y Smyrnios, 2001; Gómez et al, 2012). Sin embargo, son escasos los estudios que muestran la medición de la influencia de la familia en el proceso de gestión financiera, por lo que la influencia o el impacto del involucramiento familiar están lejos de ser claros en la literatura financiera, y más aún en el proceso de toma de decisiones.

El objetivo de la investigación de donde se derivó el presente artículo fue determinar el impacto de la influencia familiar en el negocio, sobre el proceso de gestión financiera y en la generación de valor en empresas familiares del sector textil-confección en la ciudad de Medellín y Área Metropolitana; para lo cual se realizó una encuesta a los tomadores de decisiones financieras en 100 empresas y se calculó su EVA con cifras extraídas de sus estados financieros con corte a 2015.

Este documento está conformado por cinco secciones. En la sección dos se desarrolla la base teórica dentro del contexto de la gestión financiera y los indicadores de rendimiento. En la sección tres se analiza el diseño empírico de los datos y la metodología. En la sección 4 , se presentan los principales resultados y finalmente, en la sección cinco, se discuten los hallazgos y se concluye, teniendo en cuenta las líneas de investigación futuras y las limitaciones del estudio. 


\section{Marco teórico y literatura empírica previa}

\section{Rendimiento y generación de valor en la gestión financiera}

El rendimiento se define como la medida de la productividad de los recursos comprometidos en un negocio, donde lo importante es garantizar su permanencia y crecimiento, y por ende la generación del valor para los inversionistas (García, 2009). Se afirma que las empresas buscan crecer y permanecer en el tiempo, lo cual se obtiene si logran mantener o acrecentar el patrimonio de los inversionistas. Hecho que busca medirse mediante un sinnúmero de indicadores financieros, unos con origen en la información contable, otros en la información de mercado y los indicadores basados en la metodología de la gestión del valor (Ittner y Larcker, 2003).

Permanentemente se realizan análisis sobre el desempeño de las organizaciones mediante el uso de diferentes indicadores financieros de origen contable. Los más usados en las investigaciones revisadas son los de crecimiento en ventas, en activos y en utilidades; los de eficiencia (rotación de cartera, rotación de inventarios, rotación de activos operacionales y rotación de activos totales), los de eficacia (los diferentes márgenes de utilidad y la relación EBITDA/ventas), y los de efectividad o productividad (Rendimientos sobre los activos- ROA, por sus siglas en inglés- Return On Assets y el rendimiento sobre el Patrimonio- ROE por sus siglas en inglés - Return On Equity) (Rivera y Ruiz, 2011). Estos últimos son los que se utilizan en la mayoría de los estudios.

Los indicadores de crecimiento muestran el comportamiento de las ventas, de los activos y de las utilidades a través del tiempo, lo que permite analizar si el tamaño de la empresa es creciente, decreciente o estable. Los indicadores de eficiencia miden la capacidad que tiene la empresa de producir flujos de efectivo con la menor inversión en activos. Los indicadores de eficacia miden la capacidad del negocio para producir utilidades, mediante el incremento de las ventas en mayor proporción a los costos y gastos. Los de efectividad o productividad miden la capacidad para producir utilidades teniendo en cuenta el monto de los activos, su naturaleza (los operativos o los totales) y los derechos de propiedad de los recursos utilizados (patrimonio); para determinarlos se relaciona la utilidad operativa con los activos (ROA), que mide el rendimiento de la empresa; o la utilidad neta con el patrimonio (ROE) que mide el rendimiento para los propietarios (Rivera y Ruiz, 2011).

Dentro de indicadores de desempeño financiero que utilizan información de mercado, el más usado en las investigaciones revisadas es la Q de Tobin. Aunque existen diferentes metodologías para su cálculo, suele calcularse mediante la metodología propuesta por Lang, Ofek y Stulz (1996), que hace referencia al valor de mercado de las acciones ordinarias más el valor de pasivos en libros/el valor en libros de los activos.

El indicador basado en metodologías de gestión del valor más usado es el EVA (Valor Económico Agregado), el cual surge dadas las limitaciones atribuidas a los indicadores conta- 
bles y de mercado. El EVA se muestra como una herramienta que le permite a los financieros calcular y evaluar la riqueza generada por la empresa, teniendo en cuenta el riesgo operativo (Stewart, 1991). Se calcula como la diferencia entre la UODI (utilidad operativa después de impuestos) y el costo que implica la utilización de activos (García, 2003).

Influencia familiar en el negocio sobre la generación de valor y la gestión financiera en las empresas familiares: Visión general

Influencia familiar en el negocio sobre la generación de valor y la gestión financiera en las empresas familiares: Visión general

La investigación sobre la influencia de la intervención familiar en el desempeño de las empresas familiares es cada vez mayor, pero los resultados son mixtos, especialmente para las empresas que no cotizan en bolsa (Sciascia y Mazzola, 2008). Existen diversos estudios que analizan la diferencia en los rendimientos de las empresas familiares y las no familiares. La teoría de agencia en la empresa familiar, trata de explicar por qué al interior de éstas son menores los costos de agencia, y se fundamenta en que como la propiedad y la gestión están en poder de la familia, se origina mayor eficiencia en el uso de los recursos y por ende en su rendimiento (Esparza, García y Duréndez, 2010; Maury, 2006; Cabrera, De Saá-Pérez y García, 2001). El control familiar activo se asocia con una mayor rentabilidad en comparación con las empresas no familiares, mientras que el control pasivo de la familia no afecta a la rentabilidad. Estos resultados sugieren que el control familiar reduce el problema de agencia entre propietarios y gerentes (Maury, 2006). Molina, Botero y Montoya (2017) hacen un recorrido sobre los estudios de rendimiento que se han realizado en empresas de familia y evidencian la contradicción empírica en sus resultados. Muestran que existe un grupo de autores que "señalan que las empresas de familia por tener características distintivas favorables y, concentradas la propiedad y la gestión, tienen menores costos de agencia entre propietarios y agentes, por lo tanto, obtienen un desempeño financiero superior" (p.81). Otros que concluyen "que las empresas familiares tienen un menor desempeño, debido a que éstas son mal gestionadas por sus propietarios por falta de profesionalización, el nepotismo, la falta de experiencia de los sucesores y porque el control de la empresa muy concentrado permite hacer uso indebido del poder y cerrarse a las tendencias del mercado" (p. 81). Y por último, un tercer grupo de autores que afirman que "las empresas familiares tienen igual desempeño a las empresas no familiares, dado que la estructura de capital (concentración de la propiedad), el tipo de gerente y las políticas financieras no afectan de manera directa el rendimiento. Además, que las ineficiencias generadas por las familias balancean los resultados. Los costos de agencia se ven disminuidos por la forma de la propiedad y de la gestión, sin embargo, otros costos de agencia se incrementan" (p.81).

Dado el panorama anterior, se quiso conocer cómo se da este tipo de relación en las empresas 
colombianas de un sector específico, como lo es el sector Textil-Confección en la ciudad de Medellín y su área metropolitana. Además, es importante resaltar, que los trabajos realizados sobre la relación que existe entre la influencia de la familia en el negocio y el proceso de gestión financiera son escasos; por ejemplo, Filbeck y Lee (2000) en su trabajo exploran las técnicas de gestión financiera de las empresas familiares. Examinan las empresas familiares para entender hasta qué punto utilizan técnicas de presupuestos de capital, técnicas para medir el riesgo y técnicas de gestión del capital de trabajo. Encuentran que las empresas familiares con más años de existencia, más grandes, que tienen un consejo de administración externo o un miembro no familiar en el rol de toma de decisiones financieras tienen más probabilidades que sus contrapartes más pequeñas de emplear sofisticadas técnicas de gestión financiera.

Gallo, Tápies, y Cappuyns (2004) en su investigación han identificado diferencias importantes entre las empresas familiares y las empresas no familiares. Ellos comparan las razones o lógicas financieras de los dos tipos de empresas. En términos generales, al analizar las políticas financieras implementadas en ambos tipos de empresas, las diferencias encontradas indican que las preferencias personales en cuanto al crecimiento, el riesgo y el control de la propiedad pueden ser las fuerzas impulsoras de la "lógica financiera peculiar" de las empresas familiares. Los autores concluyen que algunas de estas empresas carecen de una auténtica política de negocios a largo plazo o de un compromiso con el crecimiento y la evolución. Si la aversión al riesgo y la pérdida de control se deben a las aprehensiones o ambiciones personales del dueño-gerente, entonces ese dueño-gerente, con o sin intención, estropea las posibilidades de la compañía de competir en el futuro.

Esparza, García y Duréndez, (2010) buscaron analizar las principales diferencias de gestión financiera entre las micro, pequeñas y medianas empresas turísticas mexicanas familiares y no familiares, considerando variables como: financiación, endeudamiento, rentabilidad, crecimiento y utilización de información contable y financiera. Los resultados que obtuvieron muestran que las empresas familiares obtienen mayor rentabilidad y utilizan en menor medida la información contable y financiera que las no familiares.

Se puede notar que estos estudios buscaron comparar las prácticas financieras de las empresas familiares con las prácticas de las no familiares. Estos estudios han sido descriptivos, pero ninguno ha pretendido medir cómo impacta la influencia de la familia en el negocio el proceso de toma de decisiones financieras, cuyo objetivo si es el de esta investigación.

\section{Metodología de la investigación}

Los elementos muestrales se seleccionaron de la base de datos suministrada por el Centro de Estudios Económicos de Fenalco Antioquia. Según dicha información la población objetivo estuvo conformada por 193 empresas del sector textil y confección de la ciudad de Medellín y su área metropolitana. El valor correspondiente al tamaño de la muestra estadística obteni- 
do mediante la aplicación de la siguiente formula del método de muestreo aleatorio simple (Torres, Paz y Salazar, 2006) es de $n=97$ empresas:

$$
n=\frac{Z_{\alpha}^{2} p(1-p) N}{e^{2}(N-1)-Z_{\alpha}^{2} p(1-p)}
$$

Soporte teórico de las variables utilizadas en la medición

Todas las variables utilizadas en la medición fueron ampliamente descritas en Molina (2017), sin embargo, a continuación, se resaltarán algunos de los elementos allí desarrollados.

Variable influencia de la familia en el negocio. Después de analizar 62 estudios empíricos, como se evidencia en la tabla 1, esta variable decide medirse a través de la Escala F-PEC, propuesta y validada por Klein et al (2005), dadas las ventajas importantes que presenta dicha escala frente a la mayoría de los métodos que se han utilizado para operacionalizar el constructo influencia de la familia (Molina, 2017).

Este instrumento evalúa tres factores de la influencia de la familia en una escala continua en lugar de una escala categórica; dichos factores son el poder, la experiencia y la cultura. El poder, se entiende como la influencia que la familia tiene en la gobernanza y la gestión de la empresa; la experiencia, como el conocimiento de la información, el juicio y la intuición que viene a través de las generaciones sucesivas; y la cultura, como la alineación de los objetivos de la familia con los objetivos de la empresa.

Tabla 1

Medidas de influencia de la familia en el negocio utilizadas en estudios empíricos

\begin{tabular}{lll}
\hline Estudios & Componentes & Medida de la participación de la familia en el negocio. \\
\hline Jacquemin y De Ghellinck (1980) & Propiedad & Los accionistas mayoritarios. \\
Holderness y Sheehan (1988) & Propiedad & Porcentaje de participación en la propiedad. \\
Chaganti y Damanpour (1991) & Propiedad & Porcentaje de participación de la propiedad. \\
Daily y Dollinger (1992) & Propiedad & Gerentes relacionados con el propietario. \\
& Gestión & \\
Galve y Salas (1996) & Propiedad & Los accionistas mayoritarios.
\end{tabular}


Beehr et al. (1997)

McConaughy et al. (1998)

Lauterbach y Vaninsky (1999)

Smith y Amoako-Adu (1999)

McConaughy y Phillips (1999)

Chua et al. (1999)

Daily y Near (2000)

Gomez-Mejia

et al. (2001)

Schulze

et al. (2001)

McConaughy,

Matthews, y

Fialko (2001)
Propiedad Participación en el capital, puestos directivos, director general de la familia.

Gestión

Gobernanza

Gestión

Dirigido por el fundador o descendiente del fundador.

Sucesión

Propiedad

Estructura de la propiedad.

Gestión

Dirigido por el fundador o descendiente.

Sucesión

Propiedad

El gerente respondió que la empresa era o no familiar.

Gestión

Propiedad

Porcentaje de propiedad del negocio de miembros de la familia, número de administradores de la familia, expectati-

Gestión

Gobernanza va de que el futuro sucesor del presidente será un miembro de la familia.

Sucesión

Propiedad Participación en las decisiones operativas importantes, planes de sucesión del liderazgo influidos por miembros

Gestión de la familia.

Gobernanza

Propiedad

Apellido del propietario y editor.

Propiedad

Dos o más miembros de la familia con el mismo apellido en la empresa.

Propiedad Porcentaje de propiedad. 
Faccio, Lang y Young (2001)

Astrachan et al ( 2002)

Chrisman, Chua y Steier (2005

Anderson y Reef (2003)

Zahra (2003)

Cronqvist y Nilsson (2003)

Lee (2004)
Propiedad Monto de los votos y el capital controlado por la familia más grande.

\section{Gobernanza}

Propiedad Porcentaje de participación en la propiedad, la participación en la dirección y en el control de la empresa. La Gestión influencia de la familia sobre la Experiencia y la Cultura

Gobernanza o (F-PEC).

control

Sucesión

Propiedad Porcentaje de la propiedad, la participación de la familia en

Gestión el negocio y la intención de sucesión.

Sucesión

Propiedad

Participación en el capital fraccional de la familia fundadora y / o la presencia de miembros de la familia en la

Gobernanza dirección.

Propiedad

Porcentaje de acciones, la participación familiar en las

Gestión operaciones del negocio.

Propiedad Derechos de control (\% del total de los derechos de voto).

Gobernanza

Propiedad

Única familia controladora, miembros de la familia activos en la alta dirección, la familia involucrada (2 generacio-

Gestión nes).

Gobernanza

Sucesión 
Chua, Chrisman y Chang (2004) Propiedad

Gestión

Gobernanza

Sucesión

\section{Chrisman}

et al. (2004)

Yammeesri y Lodh (2004)

Klein et al ( 2005)

Jaskiewicz, González, Menéndez
y Schiereck (2005)

$\mathrm{Ng}(2005)$

Chrisman et al (2005)

Fernández y Nieto (2005)

Fernández y Nieto (2005)
Propiedad

Gestión

Sucesión

Propiedad

Propiedad

Gestión

Gobernanza o

control

Sucesión

Propiedad

Gobernanza

Propiedad

Gestión

Propiedad

Gestión

Gobernanza

Sucesión

Propiedad

Gestión

Gobernanza
Porcentaje de propiedad de miembros de la familia, el número de administradores de la familia, la expectativa de que el futuro presidente será miembro de la familia.

Porcentaje propiedad de la familia, el número de miembros de la familia que participan en la gestión, miembro de la familia como sucesor.

Mayores accionistas.

Porcentaje de participación en la propiedad, la participación en la dirección y en el control de la empresa. La influencia de la familia sobre el la Experiencia y la Cultura (F-PEC).

Porcentaje de participación, la participación en la dirección y La influencia de la familia Sobre el Poder, la Experiencia y la Cultura (F-PEC).

Porcentaje de propiedad del directivo.

Poder, la Experiencia y la Cultura (F-PEC)

\section{.}


Cliff y Jennings (2005)

Barontini y Caprio (2006)

Favero, Giglio, Honorati y Panunzi (2006)

Maury (2006)

Lee (2006)

Pérez-González

(2006)

Villalonga y Amit (2006)

Dyer (2006)
Propiedad

Participación de la familia a través del Poder, Experiencia

Gestión

Gobernanza o

control

Sucesión

Propiedad

Gobernanza

Propiedad

Propiedad

Gestión

Gobernanza

Propiedad

Gestión

Gobernanza

Sucesión

Gestión

Sucesión

Propiedad

Gestión

Gobernanza

Sucesión

Propiedad

Gestión

Gobernanza

\section{y Cultura (F-PEC).}

Derechos de voto directo, derechos sobre el flujo de efectivo.

La familia es el mayor accionista.

Derecho de voto $(>10 \%)$.

Fundadores familiares o descendientes tienen acciones en la empresa o están presentes en el consejo de administración.
Gerente de familia, nuevo director general estaba relacionado por sangre o matrimonio a: (a) el director general saliente, (b) el fundador, o (c) un gran accionista.

Fundador o un miembro de su familia ya sea por sangre o matrimonio es un director o accionista.
Porcentaje de propiedad de la familia o el número de miembros de la familia que ocupan puestos de dirección o de gestión. 
Miller y LeBreton-Miller (2006) Propiedad

Gestión

Gobernanza

Sucesión

Rutherford, Muse y Oswald Propiedad (2006)

Westhead y Howorth (2006)

Bennedsen et al. (2007)

Gestión

Sucesión

Chrisman, Chua, Kellermanns y Propiedad

Chang

(2007)

\section{Gestión}

Gobernanza

Sucesión

Martínez, Stöhr y

Quiroga (2007)

Sraer y Thesmar (2007)

Naldi, Nordqvist, y

Wiklund (2007)

Braun y Sharma (2007)

López -Gracia y Sánchez - Andujar (2007)

Miller et al. (2007)
Propiedad

Propiedad

Gobernanza

Propiedad

Gestión

Propiedad

Propiedad

Propiedad

Gestión

Gobernanza
La propiedad familiar (>30\%), el control de voto (>20\%), Director General de la familia, de múltiples generaciones en los negocios.

Dos o más miembros de la familia con el mismo apellido.

$50 \%$ de las acciones en propiedad de una sola familia, precepción del gerente de una empresa familiar.

Gerente de la familia, sucesión familiar.

Porcentaje de la propiedad del negocio de miembros de la familia, número de directores de la familia, expectativa de que el sucesor del presidente será un miembro de la familia.

La propiedad familiar ( $>50 \%$ de miembros de la familia en el consejo de administración)

Fundador o un miembro de la familia es un blockholder de la empresa cuando el bloque representa más del $20 \%$ de los derechos de voto.

Porcentaje de propiedad de la familia y miembros de la familia en la alta dirección.

Porcentaje de la propiedad de la familia.

$50 \%$ de las acciones en propiedad de una sola familia.

Miembros de la familia están involucrados como los principales propietarios o gerentes (nivel de propiedad y el control de la votación, las funciones de gestión).

Sucesión 
Chang et al. (2008)

Sciascia y Mazzola (2008)

Allouche

et al. (2008)

Rutherford et al (2008)

Andrés (2008)

Silva y Majluf (2008)

Randøy et al. (2009)
Propiedad

Gestión

Gobernanza

Sucesión

Propiedad

Gestión

Propiedad

Gestión

Propiedad

Gestión

Gobernanza

Sucesión

Propiedad

Gobernanza

Propiedad

Gobernanza

Propiedad

Gobernanza

Gestión

Oswald, Muse y Rutherford Propiedad (2009)

\section{Gestión}

Propiedad

Gobernanza
Porcentaje de propiedad de la empresa en manos de los miembros de la familia, el número de miembros de la familia que participan en la gestión, la intención de que el futuro presidente sea un miembro de la familia.

Porcentaje de propiedad familiar y el porcentaje de miembros de la familia en la alta dirección.

Porcentaje de propiedad de la familia, miembros de la familia en puestos de alta dirección.

Porcentaje de participación en la propiedad, la participación en la Dirección y en el control de la empresa. La influencia de la familia sobre el la Experiencia y la Cultura (F-PEC).

Los familiares tienen más del 25\% de las acciones con derecho a voto y / o están representados en el ejecutivo o el consejo de vigilancia.

Concentración de la propiedad, los miembros de la familia en el consejo de administración.

Fundador de liderazgo familiar y propiedad de la familia fundadora.

Porcentaje de propiedad de la familia, miembros de la familia en la alta dirección.

Derechos de control (> 20\% de los derechos totales de voto), y la familia es el mayor propietario. 
Kowalewski, Talavera, y Stetsyuk Propiedad (2010)

\section{Gestión}

Gobernanza

Holt et al (2010)

Minichilli et al. (2010)

Ibrahim y Samad (2011)

Sacristán- Navarro, Gómez - An- Propiedad són y Cabeza-García

Gobernanza

Gobernanza

Sucesión

Gestión
Participación de los derechos de voto de la familia, el gerente y el presidente son de la familia.

Propiedad Participación de la familia a través del Poder, Experiencia
Gestión

Propiedad La propiedad familiar, gerente familiar.

Propiedad Fracción de la participación accionaria de todos los miembros de la familia incluyendo relación de sangre y familia según la ley $(>20 \%)$.

El propietario más grande es una familia o quien tiene más del $10 \%$ de los derechos de voto.

Fuente: Construcción propia

Variable Gestión Financiera. La operacionalización de esta variable se realizó según la revisión teórica ejecutada en el capítulo 3 de la tesis doctoral de Molina (2017) y con una adaptación de 3 de las 6 dimensiones propuestas por Vera (2012) en su documento "Metodología para el análisis de la gestión financiera en pequeñas y medianas empresas (Pymes)", el cual fue producto de su tesis doctoral. Esta adaptación incluyó cambio en las preguntas y principalmente en la escala de medición, utilizándose en este estudio la escala de Likert para determinar el grado en el que se realiza la gestión financiera en una organización.

Variable Generación de Valor. La literatura empírica muestra que las medidas basadas en la contabilidad son las que se usan con mayor frecuencia para medir la generación del valor, por lo tanto, se consideran un referente para esta investigación (Rutheford et al., 2008; Dyer, 2006; Chrisman et al., 2012). Sin embargo, en este estudio se utilizó para medir la creación de valor el EVA, como indicador de ventaja competitiva, por lo que esta investigación está fundamentada en el concepto de "familiness" (Minichilli et al., 2010; Rutherford et al., 2008) además, porque este valor tiene incluido al ROA comparado con el costo de capital 
de la empresa, ya que no solo se trata de generar ROA dentro de los negocios, sino que este sea superior a sus costo de capital para poder hablar sobre un rendimiento óptimo. En lo que respecta a los datos requeridos para el cálculo de este indicador, estos se obtienen del análisis de los Balances y Estados de Resultados de cada una de las empresas que conforman la muestra, para el año 2015.

\section{Confiabilidad, validez y objetividad de la encuesta}

Para medir la confiabilidad el método que se utilizó, fue el de Cronbach $^{\mathbf{1}}$, donde el instrumento sólo requiere de una sola versión y una sola aplicación, y el cual muestra si las respuestas a los ítems del instrumento son coherentes. El resultado para este estudio fue de 0,941 , lo que indica una confiabilidad muy alta del instrumento.

La validez es el grado en que un instrumento mide la variable que se busca medir. Pueden tenerse distintos tipos de evidencia: validez de contenido, validez de criterio, validez del constructo y validez de expertos (Hernández et al., 2010).

El instrumento diseñado garantiza la validez de contenido, dada la amplia revisión teórica que se desarrolló en los capítulos 1,2,3 y 4 de Molina (2017), para garantizar el dominio de las variables y que el instrumento midiera adecuadamente todas sus dimensiones (Hernández et al., 2010).

También se usó la validez de expertos, dado que el instrumento se sometió a revisión por parte de académicos especializados ${ }^{2}$ en el tema de gerencia financiera, en empresas de familia y estadísticos, quienes hicieron valiosas observaciones en relación a la escala de medición inicialmente propuesta y al contenido del instrumento, que fueron tenidas en cuenta en la versión final del cuestionario, buscando garantizar que el instrumento de medición mida la variable en cuestión.

La validez de criterio no se hace porque no se conoce un instrumento de medición o criterio externo que pretenda medir lo mismo.

Para la validez del constructo (atributos que se usan para explicar un fenómeno) se usó el análisis de factores, método estadístico multivariado que se utiliza para determinar el número y la naturaleza de un grupo de constructos subyacentes en un conjunto de mediciones. En este análisis, se generan variables artificiales (denominadas factores) que representan constructos, que se obtienen de las variables originales y que deben ser interpretados de acuerdo a éstas. Este método es muy útil y muy usado para medir la validez del constructo (Hernández et al., 2010; Peña, 2004). Los ítems con bajas cargas (por debajo de 0,5) en todos los factores deben desecharse de la escala porque no mide realmente lo que interesa (afecta la validez del constructo).

\footnotetext{
${ }^{1}$ Véase "Metodología de la Investigación" de Hernández et al, 2010, p. 300-304. Es la medida más utilizada y la que más se ajustaba a esta investigación.

${ }^{2} 5$ expertos en finanzas y empresas de familia y 5 en estadística.
} 
La objetividad se refiere al grado en que el instrumento es permeable a la influencia de los sesgos y tendencias de los investigadores que lo administran, califican e interpretan (Hernández et al., 2010). En este caso, se garantiza la objetividad a través de la estandarización en la aplicación de la encuesta y en la evaluación de sus resultados; se dieron las mismas instrucciones y condiciones a todas las personas que lo contestaron. Además, la aplicación se hizo por personal que tiene experiencia en la recolección de datos, pues han participado en otras investigaciones y que fue capacitado adecuadamente en el uso del instrumento y en el área.

\section{Recolección de la Información}

Se utilizaron fuentes de información secundaria, como bases de datos de la Cámara de Comercio de Medellín, del Centro de Investigaciones Económicas de Fenalco Antioquia y la base de datos "Gestor Comercial y de Crédito" suministrada por la Universidad Nacional de Colombia, para obtener la información financiera de las empresas participantes en el estudio y así poder calcular el EVA, indicador de valor usado en esta investigación. La técnica de recolección de información primaria que se usó fue la encuesta, a través de un cuestionario de preguntas cerradas denominado "Impacto de la influencia de la familia en el negocio sobre el proceso de gestión financiera y la generación del valor" (Molina, 2017), el cual cumplió con todos los requisitos de confiabilidad, validez y objetividad. La información recolectada fue proporcionada por la persona (empleado, gerente o propietario) que tiene bajo su cargo la gestión de los recursos financieros de la empresa. Cuando hubo varias personas responsables de esta actividad se seleccionó aquella que posee la mayor jerarquía.

\section{Medición de variables}

La variable influencia de la familia (variable independiente) se define a través de 7 componentes que se muestran en la tabla 2.

\section{Tabla 2}

Descripción de la variable "Influencia de la Familia" para la regresión múltiple

\begin{tabular}{ll}
\hline Variable & \\
\hline P1 & Participación de la familia en el patrimonio de la empresa. \\
P2 & Participación de la familia en la junta directiva de la empresa. \\
P3 & Participación de la familia en comité de dirección. \\
P4 & La familia propietaria actual aspira a seguir siendo la propietaria en el futuro. \\
P5 & El gerente de la empresa es miembro de la familia con mayor participación en la propiedad del \\
& negocio. \\
P6 & Participación de la familia en cargos directivos. \\
P7 & Generación de la familia que hace parte de la empresa. \\
\hline
\end{tabular}

Fuente: Construcción propia. 
En esta variable, las preguntas se midieron con una escala de razón, por ejemplo, el porcentaje de participación de la familia en el patrimonio de la empresa; dicha escala debió ser categorizada y convertidas a Likert (escala ordinal) para poder calcular la correlación canónica en SPSS, como se muestra en la tabla 3:

Tabla 3

Categorización de la variable Influencia de Familia

\begin{tabular}{lll}
\hline$\%$ de participación $(\mathrm{p})$ & Categorización & Interpretación \\
\hline $\mathrm{p}<20 \%$ & 1 & Participación mínima \\
$20 \%<=\mathrm{p}<40 \%$ & 2 & Participación baja \\
$40 \%<=\mathrm{p}<60 \%$ & 3 & Participación media \\
$60 \%<=\mathrm{p}<80 \%$ & 4 & Participación alta \\
$80 \%<=\mathrm{p}<=100 \%$ & 5 & Participación muy alta \\
\hline
\end{tabular}

Fuente: Construcción propia

Como se trata de indagar sobre las características del proceso de toma de decisiones financieras de cada empresa, se ha esquematizado la variable gestión financiera en los siguientes componentes: planeación financiera, control financiero, y los parámetros financieros para la toma de decisiones de corto y largo plazo. Sus componentes se dividieron en cinco dimensiones: planeación financiera, control financiero, decisiones de inversión, decisiones de financiamiento y gestión del capital de trabajo. Dicha variable se midió utilizando la escala de Likert para determinar el grado en el que se realiza la gestión financiera en una organización; las opciones de respuesta o puntos de la escala fueron 5, donde 1 es que no se hace el proceso y 5 es que se hace en gran medida.

Los estudios previos de la participación de la familia en el negocio y el rendimiento financiero y/o generación de valor, se han basado en medidas de mercado o en medidas de contabilidad, cuyos resultados han sido mixtos; algunos investigadores han encontrado relaciones positivas, negativas y neutras (Molina, Botero y Montoya, 2017; Rutherford et al., 2008; Dyer, 2006; Schulze et al., 2003). En este estudio se utilizó para medir la generación de valor el EVA, como indicador de ventaja competitiva.

\section{Procesamiento de la información: Metodología Estadística}

El cuestionario se aplicó a la muestra en el periodo comprendido entre el 9 de febrero y el 17 de agosto de 2016. Los datos obtenidos a través del instrumento diseñado, fueron codificados en una matriz, y analizados a través de la ejecución del programa SPSS (Paquete estadístico para las Ciencias Sociales), desarrollado por la Universidad de Chicago y uno de los más utilizados en este tipo de investigaciones (Hernández et al., 2010). A continuación, se explicará 
brevemente cada uno de los análisis utilizados para estudiar las relaciones existentes entre la influencia de la familia en el negocio y su proceso de gestión financiera, y entre la influencia de la familia y la generación del valor. Para medir la primera relación se utilizó el análisis de correlación canónica y para medir la segunda relación se utilizó la regresión múltiple.

\section{Análisis de Correlación canónica y regresión múltiple}

El análisis de correlación canónica (ACC) o simplemente análisis canónico es una de las herramientas desarrolladas para estudiar la relación existente entre dos conjuntos de variables. En el análisis de regresión múltiple se mide la relación existente entre un conjunto de variables llamadas regresoras y una variable de respuesta o dependiente, por lo que se puede considerar el ACC como una generalización del modelo de regresión múltiple; el cual busca establecer la relación entre un conjunto de variables predictoras y un conjunto de variables respuesta. Con el análisis de correlación canónica se propone determinar la correlación entre una combinación lineal de las variables de un conjunto y una combinación lineal de las variables de otro conjunto. La estrategia consiste en volver al caso clásico, donde se encuentra la correlación entre pares de variables; cada una de las cuales es una combinación de las variables de los respectivos conjuntos (Díaz, 2002). Con el ACC se busca la combinación lineal U (medida por el primer conjunto de variables) y la $\mathrm{V}$ (medida por el segundo conjunto de variables), tal que la correlación entre U y V sea tan grande como se pueda. A las dos variables anteriores es lo que se denomina variables canónicas.

El interés de este estudio en particular es determinar si el conjunto de variables predictoras "Influencia de la familia en el negocio" afecta o explica el conjunto de variables respuesta "Gestión financiera". Es importante mencionar que para utilizar el escalamiento óptimo en SPSS, el investigador deberá elegir el nivel de escalamiento de las variables, antes de ser procesadas. “....al someter un sistema multivariante a un proceso de escalamiento óptimo, la cuantificación de las categorías se genera mediante transformación de las variables. El tipo de transformaciones que se utilice para cuantificar una variable específica depende de la elección por parte del usuario del nivel de escalamiento de dicha variable" (Correa, 2008, p. 33).

Cada nivel de escalamiento define una familia de transformaciones permisibles. Se utilizan transformaciones lineales para las variables escaladas a nivel numérico y transformaciones monótonamente ascendentes para las variables escaladas a nivel ordinal. Una transformación lineal de una variable consiste en multiplicar cada uno de sus valores por una constante (ponderaciones). Luego, los valores transformados serán proporcionales a los valores originales. En consecuencia, al representar los valores originales y los transformados en un plano cartesiano se forma una línea recta.

En contraste con las transformaciones lineales, cualquier transformación que genere valores transformados no proporcionales a los originales será una transformación no lineal. Si se aplica 
una transformación no lineal y se representan los valores originales frente a los transformados en un plano cartesiano no se obtendrá una línea recta. Las transformaciones monótonamente ascendentes, que se aplican al escoger el nivel de escalamiento ordinal, forman parte de las transformaciones no lineales, y se caracterizan por el hecho de que los estadísticos de orden de la variable original coinciden con los estadísticos de orden de la variable transformada (Correa, 2008). Para este estudio todas las variables fueron categorizadas como ordinales, por lo tanto, el SPSS, a través del OVERALS ${ }^{3}$, realiza una transformación de las variables monótonamente ascendente.

Al estimar la correlación canónica entre los distintos conjuntos de variables, se obtiene diversa información que se describirá más adelante, sin embargo, es importante aclarar que, si se diera una relación perfecta entre los distintos componentes, el ajuste entre los dos conjuntos de variables debería ser 2 , porque se trabajó en 2 dimensiones. Cuando el valor del ajuste da cercano a 2 esto indica que hay un ajuste adecuado entre los dos conjuntos de variables, es decir, que existe relación entre ellas, que las escalas de medición fueron adecuadas y que hubo una buena selección de esos valores. Por lo tanto, antes de proceder a calcular las variables canónicas habrá que analizar el nivel de ajuste proporcionado por el SPSS.

Además, es fundamental calcular las correlaciones intragrupales, es decir, medir el nivel de correlación entre las variables de cada conjunto con el fin de disminuir el ruido estadístico en el análisis.

\section{Coeficiente de correlación de Pearson}

Es una prueba estadística para analizar la relación entre dos variables, se simboliza con R. Se relacionan las puntuaciones recolectadas de una variable con las puntuaciones obtenidas de la otra, con los mismos participantes o casos (Hernández et al, 2010).

\section{Regresión Lineal}

Es un modelo estadístico para estimar el efecto de una variable sobre otra. Está asociada con el coeficiente $\mathrm{R}$ de Pearson. Busca predecir las puntuaciones de una variable tomando las puntuaciones de la otra variable. Entre mayor sea la correlación entre las variables (covariación), mayor será la capacidad de predicción. El modelo considera una variable como independiente y otra como dependiente, lo cual lo establece el investigador a través de sustento teórico (Hernández et al, 2010).

\footnotetext{
${ }^{3}$ En la tesis doctoral "Contribuciones al análisis multivariante no lineal" del profesor Guillermo Correa Londoño, numeral 2.7, se define formalmente el Overals o Análisis de Correlación Canónica no Lineal.
} 
Análisis inferencial para el coeficiente de correlación $R$ de Pearson

El coeficiente de correlación R puede verse como una medida numérica de que tan bien un modelo lineal representa los puntos de un diagrama de dispersión. El diagrama, sin embargo, no contiene todos los puntos posibles. Debido a que el R se calcula con base en una muestra, se espera que los valores de $\mathrm{R}$ varíen de una muestra a otra, lo que genera la pregunta de significancia de $\mathrm{R}$ : ¿Cuál es la probabilidad que la muestra aleatoria de puntos dé una correlación fuerte, cuando en realidad los puntos de la población no están correlacionados fuertemente? (Nieves y Domínguez, 2009). Se emplea @ para representar el parámetro poblacional correspondiente a $\mathrm{R}$. Con éste la significancia de $\mathrm{R}$ será tratada a través de una prueba de hipótesis del coeficiente @.

Ho: $\varrho=0$

H1: $\varrho \neq 0$.

Para realizar dicha prueba se utilizó el estadístico de prueba t de Student, para un $\alpha$ de 0,05 . Entones si $\mathrm{p}<0.05$ se rechaza Ho y $\mathrm{R}$ es confiable o significativo.

\section{Resultados}

Impacto de la influencia de la familia sobre el proceso de gestión financiera. Análisis de correlación canónica

Con las variables depuradas a través del análisis de correlación bivariada, cuyo criterio de exclusión fue: "Valores del coeficiente de correlación muestral mayores que 0,7 o menores que $-0,7$ para dos variables independientes, es una regla general que alerta sobre problemas potenciales del multicolinealidad" (Anderson, Sweeney y Williams, 2012, p. 662), se continuó con el análisis de correlación canónica para determinar el impacto que tiene la influencia de la familia sobre cada dimensión de la Gestión Financiera. Por lo tanto, se mejoraron estas condiciones de multicolinealidad a través de la reducción de las variables que estén altamente correlacionadas con otras dentro del subconjunto.

Luego, se procedió a calcular las variables canónicas por cada pareja de variables de estudio, producto de las ponderaciones y transformaciones no lineales obtenidas a través del software SPSS, y se evaluó el ajuste del modelo para determinar si esta técnica de correlación canónica se ajusta a los datos evaluados. A continuación, se muestran las ecuaciones canónicas resultantes para cada pareja de estudio y su respectivo ajuste mostrado en la tabla 4:

La Influencia de la familia vs. Planeación Financiera

$$
U_{11}=-0.388 X_{1}-0.265 X_{2}-0.455 X_{3}+0.792 X_{4}-0.532 X_{5}-0.430 X_{6}-0.472 X_{7}
$$


$V_{11}=0.692 Y_{1}+0.369 Y_{2}-0.598 Y_{3}-0.108 Y_{4}-0.183 Y_{5}-0.049 Y_{6}+0.219 Y_{7}-0.005 Y_{8}+$ $0.105 Y_{9}-0.978 Y_{10}+0.376 Y_{11}-0.128 Y_{12}$

Donde,

$\mathrm{Xi}$ : son las variables que conforman la influencia de la familia transformadas, con $\mathrm{i}=1,2, \ldots 7$

Yi: Son las variables transformadas que conforman la dimensión de planeación financiera, con $\mathrm{i}=1,2, \ldots 12$

La Influencia de la familia vs. Control Financiero

$$
\begin{gathered}
U_{12}=0.517 X_{1}+0.692 X_{2}+0.258 X_{3}-0.585 X_{4}+0.517 X_{5}+0.141 X_{6}-0.377 X_{7} \\
V_{12}=0.114 Y_{1}-0.39 Y_{2}+0.554 Y_{3}-0.07 Y_{4}+0.546 Y_{5}-0.357 Y_{6}-0.042 Y_{7}-0.001 Y_{8}- \\
0.446 Y_{9}-0.239 Y_{10}+0.162 Y_{11}-0.36 Y_{12}+0.225 Y_{13}-0.332 Y_{14}+0.293 Y_{15}+0.093 Y_{16}+ \\
0.354 Y_{17}-0.178 Y_{18}+0.245 Y_{19}+0.373 Y_{20}+0.096 Y_{21}-0.0003 Y_{22}
\end{gathered}
$$

Donde,

$\mathrm{Xi}$ : son las variables que conforman la influencia de la familia transformadas, con $\mathrm{i}=1,2, \ldots 7$

Yi: Son las variables transformadas que conforman la dimensión de control financiero, con $\mathrm{i}=1,2, \ldots .22$

La Influencia de la familia vs. Decisiones de Inversión

$$
\begin{aligned}
& U_{13}=-0.314 X_{1}+0.186 X_{2}-0.288 X_{3}+0.288 X_{4}+0.092 X_{5}+0.645 X_{6}+0.405 X_{7} \\
& V_{13}=0.21 Y_{1}-0.953 Y_{2}+0.659 Y_{3}+0.405 Y_{4}-0.634 Y_{5}+0.231 Y_{6}-0.113 Y_{7}-0.456 Y_{8}+0.28 Y_{9}+ \\
& 0.1 Y_{10}+0.37 Y_{11}+0.259 Y_{12}
\end{aligned}
$$

Donde,

$\mathrm{Xi}$ : son las variables que conforman la influencia de la familia transformadas, con $\mathrm{i}=1,2, \ldots 7$

Yi: Son las variables transformadas que conforman la dimensión de decisiones de inversión, con $\mathrm{i}=1,2, \ldots 12$

La Influencia de la familia vs. Decisiones de Financiamiento

$$
U_{14}=0.405 X_{1}+0.115 X_{2}+0.218 X_{3}+0.601 X_{4}+0.287 X_{5}-0.319 X_{6}-0.415 X_{7}
$$


$V_{14}=0.369 Y_{1}-0.6 Y_{2}+0.392 Y_{3}-0.595 Y_{4}+0.116 Y_{5}+0.05 Y_{6}-0.121 Y_{7}+0.166 Y_{8}-0.241 Y_{9}-$
$0.296 Y_{10}+0.522 Y_{11}-0.075 Y_{12}+0.071 Y_{13}+0.302 Y_{14}+0.043 Y_{15}-0.139 Y_{16}+0.326 Y_{17}-$
$0.162 Y_{18}$

Donde,

Xi: son las variables que conforman la influencia de la familia transformadas, con $\mathrm{i}=1,2, \ldots 7$

Yi: Son las variables transformadas que conforman la dimensión de decisiones de inversión, con $\mathrm{i}=1,2, \ldots 18$

$$
\begin{aligned}
& U_{15}=0.006 X_{1}-0.583 X_{2}-0.229 X_{3}-0.164 X_{4}-0.258 X_{5}+0.848 X_{6}-0.018 X_{7} \\
& V_{15}=-0.715 Y_{1}+0.337 Y_{2}+0.218 Y_{3}-0.378 Y_{4}+0.074 Y_{5}-0.172 Y_{6}+0.302 Y_{7}+0.296 Y_{8}+ \\
& 0.028 Y_{9}-0.401 Y_{10}+0.218 Y_{11}+0.745 Y_{12}-0.293 Y_{13}
\end{aligned}
$$

Donde,

$\mathrm{Xi}$ : son las variables que conforman la influencia de la familia transformadas, con $\mathrm{i}=1,2, \ldots 7$

Yi: Son las variables transformadas que conforman la dimensión de decisiones de inversión, con $\mathrm{i}=1,2, \ldots 13$

Tabla 4

Resumen de análisis. Nivel de ajuste

Influencia de la Familia

\begin{tabular}{lcc}
\multicolumn{1}{c}{ Variables } & Ajuste & Pérdida de información \\
\hline Planeación financiera & 1,669 &, 331 \\
Control financiero & 1,722 &, 278 \\
Decisiones de inversión & 1,685 &, 315 \\
Decisiones de financiamiento & 1,741 &, 259 \\
Gestión de capital de trabajo & 1,636 &, 364 \\
\hline
\end{tabular}

Fuente: Construcción propia con base en datos obtenidos en SPSS

De la tabla 4 se puede concluir que la influencia de la familia sobre el negocio tiene un buen ajuste con cada una de las dimensiones de la gestión financiera, indicando con ello que la selección de las escalas para cada variable fue adecuada, el modelo de correlación canónica se ajustó correctamente a los datos y que la perdida de información al hacer la combinación lineal es pequeña. 
Lo anterior, permite continuar con el siguiente paso de esta metodología, el cual consiste en tomar las variables canónicas y realizar a cada par su correspondiente análisis de correlación de Pearson, a través de la regresión lineal, donde la influencia de la familia siempre se consideró la variable independiente y cada dimensión de la gestión financiera se consideró como la variable dependiente. Además, se calculó el coeficiente de Determinación $\mathrm{R}^{2}$ para estimar en qué forma se ven afectadas las dimensiones de la Gestión Financiera por la Influencia de la Familia en el Negocio, cuyos resultados se muestran en la tabla 5, y se realizaron las pruebas de hipótesis para probar la significancia de R.

Tabla 5

Resumen de los Coeficientes de correlación y de los coeficientes de determinación "Influencia de la familia vs. Gestión Financiera"

Variable Independiente

Influencia de la Familia

$\begin{array}{ccc}\text { Variables Dependientes } & \mathrm{R} & \mathrm{R} 2 \\ \text { Planeación Financiera } & 0,687 & 0,473 \\ \text { Control Financiero } & 0,671 & 0,45 \\ \text { Decisiones de Inversión } & 0,695 & 0,483 \\ \text { Decisiones de Financiamiento } & 0,778 & 0,606 \\ \text { Gestión del capital de trabajo } & 0,657 & 0,432\end{array}$

Fuente: Construcción propia con base en resultados obtenidos en SPSS

En la tabla 5 se observa una relación positiva media $(0.687)$ entre la influencia de la familia y la planeación financiera. Además, el coeficiente de determinación $\mathrm{R}^{2}$ está indicando que la influencia de la familia explica el 47,3\% la variación en la planeación financiera de la empresa quedando un valor pendiente $(52.7 \%)$ por ser explicado con otras variables de estudio.

Esto evidencia que la planeación dentro de los negocios depende además de otros factores externos a la familia. Podría aseverarse que allí influyen otros elementos relevantes como la profesionalización de los encargados de pensar y planear el negocio y de su cultura organizacional. Allí lo que prevalece es la visión y las proyecciones futuras que se tengan para la empresa.

También se puede analizar que existe una relación positiva media entre la influencia de la familia y el control financiero de la empresa $(0,671)$, es decir, que la primera variable explica al control financiero en un $45 \%$. Es lógico pensar que mientras mayor sea la participación de la familia dentro de los negocios, mayor será el control ejercido por ellos, sin embargo, queda pendiente un $55 \%$ de la variable control financiero que es explicada por otro tipo de elementos. Por mucho esfuerzo que hagan los miembros de la familia por controlar financieramente el 
negocio, habrá situaciones que se salgan de sus manos, como, por ejemplo, el garantizar que los directivos externos hagan un uso transparente de la información confidencial para el negocio, que se consideren dentro de los indicadores a medir factores externos como la competencia y los clientes, que le dediquen el tiempo suficiente a la planeación de la empresa, como paso esencial a la hora de gestionarla. Por lo tanto, serán este tipo variables las que expliquen el $55 \%$ del control financiero, porcentaje que no es explicado por la influencia de la familia.

Se evidencia que la influencia de la familia en el negocio impacta en un 0,695 la subdimensión decisiones de inversión, es decir, que existe una relación positiva media entre estos grupos de variables. El R2 indica que la primera variable explica en un 48,3\% a la segunda variable.

Se evidenció en esta investigación, que el uso de herramientas financieras para la toma de decisiones de inversión y para su posterior evaluación se da en un nivel bajo medio (calificación entre 2,01 y 3); que la herramienta más usada por los gerentes para tomar este tipo de decisiones es su intuición y experiencia en el negocio, factores que no dependen de la familia propietaria como tal, sino de la falta de educación financiera en los tomadores de decisiones. Se notó en el trabajo de campo, que poco se aplica en la mayoría de los negocios, la teoría financiera para la toma de decisiones de inversión, impartida en las universidades. Elemento que podría aportar a la explicación del 51,7\% que resta para comprender en la totalidad la subdimensión decisiones de inversión.

De la tabla 5 también se puede inferir que existe una relación positiva considerable entre la influencia de la familia en el negocio y la toma de decisiones de financiamiento, pues el coeficiente de correlación entre estas dos variables es del 0,778, y su nivel de explicación es del 60,6\%.

Existen factores en este tipo de decisiones que no pueden ser controlados por la influencia de la familia, como son la oferta de créditos con facilidades de pago, por parte de instituciones financieras para este tipo de negocios, además de poca cultura dentro de las organizaciones para el uso de alternativas de financiamiento que se aparten de las tradicionales, como son los préstamos y la autofinanciación con recursos propios; también se da muy poco el uso de herramientas para calcular y usar el costo del patrimonio y del capital para la toma de decisiones, lo que conllevaría a pensar que esto en parte se puede explicar por la falta de educación financiera en los tomadores de decisiones. Elementos todos que pudieran contribuir con la explicación del 39,4\% de las decisiones de financiamiento que no puede ser explicada por la influencia de la familia.

El coeficiente de correlación para las variables influencia de la familia y gestión del capital de trabajo fue de 0,657 y su R2 es del 43,2\%. El resto de la variable, el 56,8\% puede ser explicada por factores externos que afectan este tipo de gestión y que muy posiblemente no son influidos por la participación de la familia en el negocio, como puede ser la capacidad de negociación con el proveedor (posición dominante por parte de éste) y ciertas condiciones 
del mercado que van marcando la pauta de la gestión de inventarios, de cuentas por cobrar y del efectivo, situaciones que en ocasiones llevan a la insolvencia del negocio y que inciden en el comportamiento del mismo. Además de lo anterior, es fundamental que se conozcan las técnicas de administración que tributan a la gestión eficiente del KT, deficiencia que se detectó al interior de las empresas de la muestra, las cuales en su mayoría no gestionan adecuadamente el efectivo.

Se realizaron las pruebas de significancia para todos los R, y como P siempre resultó ser un valor menor al $0.05(\alpha)$ se infiere que hay evidencia suficiente para rechazar la hipótesis nula y, por ende, los coeficientes de correlación hallados son significativos y confiables.

Impacto de la influencia de la familia sobre la generación del valor. Análisis de Regresión Múltiple

Con el objetivo de estimar la correlación que existe entre la variable "Influencia de la familia sobre el negocio" y el "Eva", se realizó un análisis de Regresión Múltiple con las variables presentadas en la tabla 3 , donde las $P$ son las variables que componen la influencia de la familia (variables independientes) y el EVA es la variable dependiente.

Se corrió un modelo de Regresión Múltiple utilizando el método "Hacia atrás" con el fin de observar la significancia de cada variable independiente $\mathrm{P}_{\mathrm{i}}$ en la relación total del modelo. Este método introduce todas las variables al comienzo y va retirando paso a paso las variables menos significativas, con base a un valor $P$ superior a 0,05 . Como criterio estadístico para retirar del modelo las variables no significativas se utilizó la prueba F con un nivel de significancia del 10 por ciento, quedando únicamente como variables significativas P5 y P6, es decir, el gerente de la familia es miembro de la familia con mayor participación en la propiedad del negocio y la participación de la familia en cargos directivos.

En la tabla 6 se observa la evolución que tuvo el coeficiente de correlación a medida que se fueron retirando las variables no significativas. Igualmente, se observa que en la sexta iteración el $\mathrm{R}$ fue de 0,425 , lo cual, indica una correlación débil cercana a media (Hernández et al, 2010, p. 312). Además, el coeficiente de determinación corregido $\mathrm{R}^{2}$ indica una explicación del Eva de tan solo el 15.3\%. Lo anterior, evidencia que el modelo de regresión que se obtiene de la relación del EVA con la Influencia de la Familia en el Negocio no es significativo para predecir el comportamiento del EVA, ya que, queda un $85 \%$ de información de esta variable que no puede ser explicada por las variables significativas P5 y P6. Lo anterior, genera la motivación para abrir una línea de investigación enfocada a determinar qué otras variables explican el 85\% del comportamiento del EVA que no se pudo estimar en este modelo. 
Tabla 6

Evolución de Coeficiente de Correlación Regresión Múltiple, variables influencia de la familia vs EVA

\begin{tabular}{|c|c|c|c|c|c|c|}
\hline \multicolumn{7}{|c|}{ Resumen del modelog } \\
\hline \multicolumn{2}{|c|}{ Modelo } & \multirow{2}{*}{$\begin{array}{c}\mathrm{R} \\
, 455^{\mathrm{a}}\end{array}$} & \multirow{2}{*}{$\begin{array}{c}\text { R cuadrado } \\
, 207\end{array}$} & \multirow{2}{*}{$\begin{array}{c}\text { R cuadrado } \\
\text { corregida }\end{array}$} & \multirow{2}{*}{$\begin{array}{c}\begin{array}{c}\text { Error típ. de la } \\
\text { estimación }\end{array} \\
\$ 5.041 \mathrm{E} 8\end{array}$} & \multirow[t]{2}{*}{ Durbin-Watson } \\
\hline dimension0 & 1 & & & & & \\
\hline & 2 &, $455^{\mathrm{b}}$ & ,207 & ,121 & \$4.995E8 & \\
\hline & 3 &, $454^{\mathrm{c}}$ & ,206 &, 135 & $\$ 4.955 \mathrm{E} 8$ & \\
\hline & 4 &, $452^{\mathrm{d}}$ & ,204 &, 148 & $\$ 4.916 \mathrm{E} 8$ & \\
\hline & 5 &, $447^{\mathrm{e}}$ & 199 &, 158 & $\$ 4.888 \mathrm{E} 8$ & \\
\hline & 6 &, $425^{\mathrm{f}}$ &, 180 &, 153 & $\$ 4.904 \mathrm{E} 8$ & 2,288 \\
\hline \multicolumn{7}{|c|}{ a. Variables predictoras: (Constante), P7, P2, P1, P6, P3, P5, P4 } \\
\hline \multicolumn{7}{|c|}{ b. Variables predictoras: (Constante), P7, P2, P1, P6, P3, P5 } \\
\hline \multicolumn{7}{|c|}{ c. Variables predictoras: (Constante), P7, P2, P6, P3, P5 } \\
\hline \multicolumn{7}{|c|}{ d. Variables predictoras: (Constante), P2, P6, P3, P5 } \\
\hline \multicolumn{7}{|c|}{ e. Variables predictoras: (Constante), P2, P6, P5 } \\
\hline \multicolumn{7}{|c|}{ f. Variables predictoras: (Constante), P6, P5 } \\
\hline \multicolumn{7}{|c|}{ g. Variable dependiente: EVA } \\
\hline
\end{tabular}

Fuente: Construcción propia con base en resultados obtenidos en SPSS

Finalmente, se puede concluir con base a los datos de la tabla 7 que dentro del 15\% explicado por el modelo resultante en la iteración 6 ( $\mathrm{R}^{2}$ ajustado), se evidencia que la variable P5 tiene un efecto inverso sobre el EVA, es decir, que cuando el gerente hace parte de la familia propietaria el valor del EVA se disminuye. Caso contrario con el efecto de la variable P6 que presenta una relación directa con el EVA, por lo tanto, es beneficioso para el EVA que la familia propietaria participe en los cargos directivos del negocio. Lo anterior, permite inferir que en las empresas de familia es recomendable la presencia de los miembros de la familia en cargos directivos en pro de incrementar la generación del valor, con la salvedad de que el Gerente no debe ser miembro de la familia. 
Tabla 7

Coeficientes del modelo significativo.

\begin{tabular}{|c|c|c|}
\hline \multicolumn{3}{|c|}{ Coeficientes } \\
\hline & & Coeficientes no estandarizados \\
\hline \multicolumn{2}{|c|}{ Modelo } & \\
\hline \multirow{3}{*}{6} & (Constante) & $-234706836,178$ \\
\hline & P5 & $-323217875,220$ \\
\hline & P6 & 567971401,176 \\
\hline
\end{tabular}

Fuente: Construcción propia en SPSS

\section{Conclusiones}

Se midió el impacto de la influencia de la familia sobre cada una de las dimensiones de la variable de gestión financiera, a través de un método avanzado de estadística multivariada, la correlación canónica. Se encontró que la influencia de la familia en el negocio impacta positivamente, pero en un nivel medio, a cuatro dimensiones de la gestión financiera como son: la planeación financiera, el control financiero, las decisiones de inversión y la gestión del capital de trabajo, dándoles una explicación que va desde el 42,3\% hasta el 47,3\%. Las decisiones de financiamiento son la dimensión que más impacta la influencia de la familia, ya que esta variable explica este tipo de decisiones en un $60,6 \%$ y existe una correlación considerable entre ellas.

El impacto de la influencia de la familia sobre la generación de valor se midió a través de una regresión múltiple. Se encuentra que el impacto causado por la influencia de la familia sobre el EVA es positivo pero débil, y cuya explicación de la variable sólo es del 15,3\%. Es importante resaltar que, según estos resultados, es recomendable la presencia de los miembros de la familia en cargos directivos en pro de incrementar la generación del valor, con la salvedad de que el Gerente no debería ser miembro de la familia.

En esta investigación se encuentra que la mayoría de las empresas del sector textil y confección de Medellín y su área metropolitana no generan valor económico para sus inversionistas, por el contrario, lo destruyen. Lo que va en contravía de los hallazgos de diferentes autores como: Santana y Cabrera, 2001; McConaughy et al., 2001; Miller y Le Breton, 2006; Anderson y Reeb, 2003; Barontini y Caprio, 2006; Maury, 2006; Lee, 2006; Chrisman, Chua, Kellermanns y Chang, 2007; Martínez, Stohr y Quiroga, 2007; Sraer y Thesmar, 2007; Silva y Majluf, 2008; Andrés, 2008; Allouche, Amann, Jaussaud y Kurashina, 2008; Randøy, Dibrell y Craig; 2009; Bjuggren y Palmberg , 2010; Esparza et al., 2010; González et al, 2012; quie- 
nes señalan en sus estudios que las empresas de familia por tener características distintivas favorables, y concentradas la propiedad y la gestión, tienen menores costos de agencia entre propietarios y agentes, por lo tanto, obtienen un desempeño financiero superior.

Lo anterior puede explicarse porque en el contex to Colombiano y en general en las economías latinoamericanas, por su idiosincrasia, además de no darse las condiciones para que la teoría de agencia entre agente y propietario se cumpla, las empresas no poseen algunas de las características descritas en estudios anteriores, realizados en otros contextos, como son: los miembros de la familia toman mejores decisiones de inversión y de distribución de dividendos, tienen mejores motivaciones e incentivos económicos por parte de los directivos y propietarios y menores niveles de costos contractuales (Kotey, 2005).

Los resultados de este estudio parecen reforzar los hallazgos de autores como: Westhead y Howorth, 2006; Sacristán, Gómez y Cabeza, 2011; Schulze, Lubatkin y Dino, 2003; Jaskiewicz, González, Menéndez y Schiereck, 2005; Minichilli, Corbetta y MacMillan, 2010; Chrisman, Chua y Litz, 2004; Braun y Sharma, 2007; Laitinen, 2008; Miller, Le Breton, Lester y Cannella, 2007. Quienes afirman que no existe diferencia en el desempeño financiero de las empresas familiares y no familiares, dado que la concentración de la propiedad y el tipo de gerente no afectan de manera directa el rendimiento, las ineficiencias generadas por las familias balancean los resultados y mientras los costos de agencia entre propietarios y agentes se ven disminuidos, otros costos de agencia se incrementan. Además, la lógica de la creación de valor en las empresas familiares difiere a la lógica de las no familiares.

Se recomiendan para futuras investigaciones en este campo, aquellos estudios que solucionen algunas de las limitaciones de la presente investigación, tales como incluir análisis métricos de la revisión por países o regiones donde se realizaron las investigaciones, por idiomas, por años o periodos de publicación entre otros datos estadísticos que puedan resultar de interés para tener una visión más completa de este tipo de organizaciones y comprender sus tendencias; la realización de análisis empíricos consolidados que permitan reconocer las características de las empresas de familia de acuerdo a otras variables, tales como el tamaño de las empresas, comparativos entre industrias y la generación de nuevos y diferentes indicadores financieros que permitan dar cuenta de las potencialidades de las empresas familiares.

Otros elementos fundamentales a considerar en el análisis de las decisiones financieras son las condiciones del entorno y la formación profesional de los tomadores de decisiones. Se sugiere que mientras mayor sea la formación académica y el conocimiento del mercado, mejor será el nivel de gestión en estas empresas. Pero como no existe evidencia empírica al respecto, esta relación podría ser una futura línea de investigación. Se requieren de nuevas propuestas académicas que enriquezcan la literatura alrededor de estas empresas, dada su complejidad y sus nuevas realidades.

Desarrollar un trabajo investigativo que identifique cuáles son los otros factores que termi- 
nan de explicar las dimensiones de la gestión financiera y que expliquen de manera profunda la generación del valor en estas organizaciones, no solo considerando las variables contables y financieras, sino la concepción que dichas organizaciones tienen alrededor de esta variable.

\section{Referencias}

Allouche, J., Amann, B., Jaussaud, J. y Kurashina, T. (2008). The Impact of Family Control on the Performance and Financial Characteristics of Family versus Nonfamily Businesses in Japan: A Matched-Pair Investigation. Family Business Review, 21(4), 315-329. DO I: $10.1177 / 08944865080210040104$

Anderson, R. y Reeb, D. (2003). Founding-family ownership and firm performance: evidence from the S\&P 500. Journal of Finance, 58 (3), 1301-1327. DOI: 10.2307/3094581

Anderson, D., Sweeney, D. y Williams, T. (2012). Estadística para negocios y economía. (11 ${ }^{\mathrm{a}}$ Ed.). México: Cengage Learnig Editores, S.A.

Andres, C. (2008). Large shareholders and firm performance: An empirical examination of founding-family ownership. Journal of Corporate Finance, 14, 431-445.

Astrachan, J., Klein, S. B. y Smyrnios, K. X. (2002). The F-PEC scale of family influence: a proposal for solving the family business definition problem. Family business review, 15(1), 45-58. https://doi.org/10.1111/j.1741-6248.2002.00045.x

Barontini, R. y Caprio, L. (2006). The effect of family control on firm value and performance: Evidence from continental Europe. European Financial Management, 12, 689723. https://ssrn.com/abstract=675983or http://dx.doi.org/10.2139/ssrn.675983

Beehr, T. A., Drexler, J. A. y Faulkner, S. (1997). Working in small family businesses: empirical comparisons to non-family businesses. Journal of Organizational Behavior, 18(3), 297-312. https://www.jstor.org/stable/3100146

Bennedsen, M., Nielsen, K., Pérez-González, F. y Wolfenzon, D. (2007). Inside the family firm: The role of families in succession decisions and performance. Quarterly Journal of Economics,122, 647-691.https://doi.org/10.1162/qjec.122.2.647

Bjuggren, P.-O. y Palmberg, J. (2010). The impact of vote differentiation on investment performance in listed family firms. Family Business Review, 23, 327-340. https://doi. org/10.1177/0894486510379001

Braun, M., y Sharma, A. (2007). Should the CEO also be chair of the board? An empirical examination of family-controlled public firms. Family Business Review, 20, 111-126. https://doi.org/10.1111/j.1741-6248.2007.00090.x

Cabrera, K., De Saá-Pérez, P. y García-Almeida, D. (2001). The succession process from a resource- and knowledge-based view of the family firm. Family Business Review. 14(1), 37-47. https://doi.org/10.1111/j.1741-6248.2001.00037.x 
Chaganti, R., y Damanpour, F. (1991). Institutional ownership, capital structure, and firm performance. Strategic Management Journal, 12,479-491. https:/www.jstor.org/stable/2486521

Chang, E. P. C., Chrisman, J. J., Chua, J. H., y Kellermanns, F. W. (2008). Regional economy as a determinant of the prevalence of family firms in the United States: A preliminary report. Entrepreneurship: Theory and Practice, 32, 559-573. https://doi.org/10.1111/ j.1540-6520.2008.00241.x

Chrisman, J., Chua, J. y Litz, R. A. (2004). Comparing the agency costs of family and nonfamily firms: Conceptual issues and exploratory evidence. Entrepreneurship Theory and Practice, 28, 335-344. https://doi.org/10.1111/j.1540-6520.2004.00049.x

Chrisman, J. J., Chua, J. H. y Steier, L. (2005). Sources and consequences of distinctive familiness: An introduction. Entrepreneurship Theory and Practice, 29(3), 237-247. https://doi.org/10.1111/j.1540-6520.2005.00080.x

Chrisman, J., Chua, J., Kellermanns, F. W. y Chang, E. P. C. (2007). Are family manager's agents or stewards? An exploratory study in privately held family firms. Journal of Business Research, 60, 1030-1038. https://doi.org/10.1016/j.jbusres.2006.12.011

Chrisman, J. J., Chua, J. H., Pearson, A. W. y Barnett, T. (2012). Family involvement, family influence, and family-centered non-economic goals in small firms. Entrepreneurship theory and practice, 36(2), 267-293. https://doi.org/10.1111/j.1540-6520.2010.00407.x

Chua, J., Chrisman, J. y Sharma,P.(1999). Defining the family business by behavior. Entrepreneurship theory and practice, 23(4), 19-40.https://doi.org/10.1177/104225879902300402

Chua, J., Chrisman, J. y Chang, E. P. C. (2004). Are family firms born or made? An exploratory investigation. Family Business Review, 17, 37-54. https://doi.org/10.1111/j.17416248.2004.00002.x

Cliff, J. E. y Jennings, P. D. (2005). Commentary on the Multidimensional Degree of Family Influence Construct and the F-PEC Measurement Instrument. Entrepreneurship Theory and Practice, 29(3), 341-347. https://doi.org/10.1111/j.1540-6520.2005.00087.x

Correa, G. (2008). Contribuciones al análisis multivariante no lineal. (Tesis Inédita de doctorado). Universidad de Salamanca Departamento de Estadística, España.

Cronqvist, H. y Nilsson, M. (2003). Agency Costs of Controlling Minority Shareholders. Journal of Financial \& Quantitative Analysis, 38(4), 695-719. https://www.jstor.org/ stable/4126740

Daily, C. M. y Dollinger, M. J. (1992). An empirical examination of ownership structure in family and professionally managed firms. Family Business Review, 5, 117-136. https:// doi.org/10.1111/j.1741-6248.1992.00117.x

Daily, C. M. y Near, J. P. (2000). CEO satisfaction and firm performance in family firms: Divergence between theory and practice. Social Indicators Research, 51 (2), 125-170. https://link.springer.com/article/10.1023/A:1007099301884

Díaz, L. (2002). Estadística Multivariada: inferencia y métodos. Bogotá: Panamericana Formas e Impresos. 
Dyer, W. G. (2006). Examining the "family effect" on firm performance. Family Business Review, 19, 253-273. https://ssrn.com/abstract=950405 or http://dx.doi.org/10.1111/ j.1741-6248.2006.00074.x

Esparza, J., García, D. y Duréndez, A. (2010). Diferencias de gestión financiera entre empresas familiares y no familiares del sector turístico mexicano. Actualidad Contable FACES, 20, 29-48. https://biblat.unam.mx/hevila/ActualidadcontableFACES/2010/vol13/no20/3. pdf

Faccio, M., Lang, L. H. y Young, L. (2001). Dividends and expropriation. American Economic Review, 91, 54-78. https://www.jstor.org/stable/2677898

Favero, C. A., Giglio, S., Honorati, M. y Panunzi, F. (2006). The performance of Italian family firms. ECGI working paper series in finance 127/2006.

Fernández, Z. y Nieto, M. J. (2005). Internationalization strategy of small and mediumD sized family businesses: some influential factors. Family Business Review, 18(1), 77-89. https://doi.org/10.1111/j.1741-6248.2005.00031.x

Filbeck, G., y Lee, S. (2000). Financial management techniques in family businesses. Family Business Review, 13(3), 201-216. https://doi.org/10.1111/j.1741-6248.2000.00201.x Gallo, M., Tàpies, J. y Cappuyns, K. (2004). Comparison of family and nonfamily business: Financial logic and personal preferences. Family Business Review, 17(4), 303-318. https://doi.org/10.1111/j.1741-6248.2004.00020.x

Galve, C. y Salas, V. (1996). Ownership structure and firm performance: Some empirical evidence from Spain. Managerial and Decision Economics, 17(6), 575-586. https://www. jstor.org/stable/2487952

García, O. (2003). Valoración de Empresas, Gerencia de Valor y EVA. Cali: Prensa Moderna Impresores S.A.

García, O. (2009). Administración Financiera: Fundamentos y Aplicaciones. (4a Ed.) Cali: Prensa Moderna Impresores S.A.

Gómez, G., López, M., Betancourt, J. y Millán, J. (2012). Estudio sobre el desempeño de las empresas familiares colombianas que cotizan en la bolsa de valores, frente a las empresas no familiares. Entramado 8, 28-42. https://revistas.unilibre.edu.co/index.php/entramado/ article/view/3413

Gómez-Mejía, L. R., Núnez-Nickel, M., y Gutierrez, I. (2001). The role of family ties in agency contracts. Academy of management Journal, 44(1), 81-95. https://www.jstor.org/ stable/3069338

González, M., Guzmán, A., Pombo, C y Trujillo, M. (2012). Family firms and financial performance: The cost of growing. Emerging Markets Review. 13, 626-649. https://ideas. repec.org/p/uac/somwps/032.html

Hernández, R., Fernández, C. y Baptista, P.(Ed. 5). (2010). Metodología de la Investigación. México: Mc Graw Hill.

Holderness, C., y Sheehan, D. (1998). The role of majority shareholders in publicly held corporations: An exploratory analysis. Journal of Financial Economics, 20,317-346. https:// 
doi.org/10.1016/0304-405X(88)90049-9

Holt, D. T., Rutherford, M. W., y Kuratko, D. F. (2010). Advancing the field of family business research: Further testing the measurement properties of the F-PEC. Family Business Review, 23(1), 76-88. DOI: 10.1177/0894486509349943

Ibrahim, H. y Samad, F. (2011). Corporate governance mechanisms and performance of public-listed family-ownership in Malaysia. International Journal of Economics and Finance, 3, 105-115. https://pdfs.semanticscholar.org/897f/22ef22a8fa2332421c1b94b93ed7e30b9ea0.pdf

Ittner, C. y Larcker, D. (2003). Coming up short on nonfinancial performance measurement. Harvard business review, 81(11), 88-95. https://pubmed.ncbi.nlm.nih. gov/14619154/

Jacquemin, A., y de Ghellinck, E. (1980). Familial control, size and performance in the largest French firms. European Economic Review, 13(1), 81-91. https://EconPapers.repec. org/RePEc:eee:eecrev:v:13:y:1980:i:1:p:81-91

Jaskiewicz, P., González, V. M., Menéndez S. y Schiereck, D. (2005). Long-run IPO performance analysis of German and Spanish family-owned businesses. Family Business Review, 18, 179-202. https://doi.org/10.1111/j.1741-6248.2005.00041.x

Klein, S. B., Astrachan, J. H. y Smyrnios, K. X. (2005). The F-PEC Scale of Family Influence: Construction, Validation, and Further Implication for Theory. Entrepreneurship Theory and Practice, 29(3), 321-339. https://doi.org/10.1111/j.1540-6520.2005.00086.x

Kotey, B. (2005). Goals, management practices, and performance of family SMEs. International Journal of Entrepreneurial Behaviour and Research. 11(1), 3- 24. https://ssrn. com/abstract $=1500923$

Kowalewski, O., Talavera, O. y Stetsyuk, I. (2009). Influence of family involvement in management and ownership on firm performance: Evidence from Poland. Family Business Review. 23, 45-59. https://doi.org/10.1177/0894486509355803

Laitinen, E. (2008). Value drivers in Finnish family-owned firms: profitability, growth and risk. International Journal Accounting and Finance. 1(1), 1- 41. https://EconPapers. repec.org/RePEc:ids:intjaf:v:1:y:2008:i:1:p:1-41

Lang, L., Ofek, E. y Stulz, R. (1996). Leverage, investment, and firm growth. Journal of financial Economics, 40(1), 3-29. https://doi.org/10.1016/0304-405X(95)00842-3

Lee, J. (2004). The effects of family ownership and management on firm performance. SAM Advanced Management Journal, 69(4), 46-53. https://www.researchgate.net/publication/285024669_The_effects_of_family_ownership_and_management_on_firm_performance

Lee, J. (2006). Family firm performance: further evidence. Family Business Review, 19 (2), 103-114. https://doi.org/10.1111/j.1741-6248.2006.00060.x

Lauterbach, B. y Vaninsky, A. (1999). Ownership structure and firm performance: Evidence from Israel. Journal of Management and Governance, 3, 189-201. https://link.springer. com/article/10.1023\%2FA\%3A1009990008724 
López Gracia, J. y Sánchez Andújar, S. (2007). Financial structure of the family business: evidence from a group of small Spanish firms. Family Business Review, 20(4), 269287. https://doi.org/10.1111/j.1741-6248.2007.00094.x

Martínez, J. I., Stohr, B. S. y Quiroga, B. F. (2007). Family Ownership and Firm Performance: Evidence from Public Companies in Chile. Family Business Review, 20(2), 8394. https://doi.org/10.1111/j.1741-6248.2007.00087.x

Maury, B. (2006). Family ownership and firm performance: Empirical evidence from Western European corporations. Journal of Corporate Finance, 12, 321-341). https://doi. org/10.1016/j.jcorpfin.2005.02.002

McConaughy, D., Walker, M. C., Henderson, G. V., y Mishra, C. S. (1998). Founding family controlled firms: Efficiency and value. Review of Financial economics, 7(1), 1-19. https://doi.org/10.1016/S1058-3300(99)80142-6

McConaughy, D. L. y Phillips, G. M. (1999). Founders versus descendants: The profitability, efficiency, growth characteristics and financing in large, public, founding-family-controlled firms. Family Business Review, 12(2), 123-131. https://doi.org/10.1111/j.17416248.1999.00123.x

McConaughy, D., Mattews, C. y Fialko, A. (2001). Founding family controlled firms: Performance, Risk, and value. Journal of Small business management, 39 (1), 31-49. https:// doi.org/10.1111/0447-2778.00004

Miller, D., y Le Breton-Miller, I. (2006). Family governance and firm performance: Agency, stewardship and capabilities. Family Business Review, 19 (1), 73-87. https://ssrn.com/ abstract=889417 or http://dx.doi.org/10.1111/j.1741-6248.2006.00063.x

Miller, D., Le Breton-Miller, I., Lester, R. y Cannella Jr., A. (2007). Are family firms really superior performers? The Journal of Corporate Finance, 13 (5), 829-858. DOI: 10.1016/j. jcorpfin.2007.03.004

Minichilli,A., Corbetta, G., y MacMillan, I. C. (2010). Top management teams in family controlled companies: 'Familiness', 'faultlines', and their impact on financial performance. Journal of Management Studies, 47,205-222. doi: 10.1111/j.1467-6486.2009.00888.x

Molina, P. (2017). Análisis sectorial del impacto de la incidencia familiar en el negocio sobre el proceso de gestión financiera y la generación de valor en empresas familiares. Sector textil- confección en Medellín y área metropolitana. (Tesis Inédita de doctorado). Universidad Nacional de Colombia, Facultad de Minas, Colombia.

Molina, P., Botero, S. y Montoya, A. (2017). Estudios de rendimiento en empresas de familia. Una nueva perspectiva. Estudios Gerenciales, 33, 76-86. https://doi.org/10.1016/j. estger.2016.10.009

Naldi, L., Nordqvist, M. K. S. y Wiklund, J. (2007). Entrepreneurial orientation, risk taking and performance in family firms. Family Business Review, 10, 33-47. https://doi. org/10.1111/j.1741-6248.2007.00082.x

Nieves, A y Domínguez, F. (2009). Probabilidad y Estadística para ingeniería, un enfoque moderno. México: McGraw Hill Interamericana. 
$\mathrm{Ng}$, C. (2005). An empirical study on the relationship between ownership and performance in a family-based corporate environment. Journal of Accounting. Auditing and Finance, 20, 121-146. DOI: 10.1177/0148558X0502000202

Oswald, S. L., Muse, L. A., y Rutherford, M. W. (2009). The influence of large stake family control on performance: Is it agency or entrenchment?. Journal of Small Business Management, 47,116-135. https://doi.org/10.1111/j.1540-627X.2008.00264.X

Pérez-González, F. (2006). Inherited control and firm performance. The American economic review, 1559-1588. https://www.aeaweb.org/articles?id=10.1257/aer.96.5.1559

Poutziouris, P. (2001). The views of family companies on venture capital: Empirical evidence from the UK small to medium-side enterprising economy. Family Business Review, 14(3), 227. DOI: 10.1111/j.1741-6248.2001.00277.x

Randøy, T., Dibrell, C. y Craig, J. (2009). Founding family leadership and industry profitability. Small Business Economics, 32,397-407. https://link.springer.com/article/10.1007/ s11187-008-9099-9

Rivera, J. y Ruiz, D. (2011). Análisis del desempeño financiero de empresas innovadoras del sector alimentos y bebidas en Colombia. Pensamiento \& Gestión, (31), 109-136. http:// rcientificas.uninorte.edu.co/index.php/pensamiento/article/view/3661/4990

Rutherford, M. W., Muse, L. A., y Oswald, S. L. (2006). A new perspective on the developmental model for family business. Family Business Review, 19(4), 317-333. https://doi. org/10.1111/j.1741-6248.2006.00079.x

Rutherford, M., Kuratko, D. y Holt, D. (2008). Examining the link between "familiness" and performance: can the F $\square$ PEC untangle the family business theory jungle? Entrepreneurship theory and practice, 32(6), 1089-1109. https://doi.org/10.1111/j.15406520.2008.00275.x

Sacristán-Navarro, M., Gómez-Ansón, S., y Cabeza-García, L. (2011). Family ownership and control, the presence of other large shareholders, and firm performance: Further evidence. Family Business Review, 24, 71-93. https://doi.org/10.1177/0894486510396705

Santana, D. y Cabrera-Suárez, K. (2001). Comportamiento y resultados de las empresas cotizadas familiares versus no familiares. XI Congreso Nacional de ACEDE.

Schulze, W. S., Lubatkin, M. H., Dino, R. N. y Buchholtz, A. K. (2001). Agency relationships in family firms: Theory and evidence. Organization science, 12(2), 99-116. https:// www.jstor.org/stable/3086050

Schulze, W., Lubatkin, M. y Dino, R. (2003). Toward a theory of agency and altruism in family firms. Journal Business Venturing, (18), 473- 490. https://EconPapers .repec.org/ RePEc:eee:jbvent:v:18:y:2003:i:4:p:473-490

Sciascia, S. y Mazzola, P. (2008). Family involvement in ownership and management: Exploring nonlinear effects on performance. Family Business Review, 21, 331-345. https:// doi.org/10.1177/08944865080210040105

Silva, F. y Majluf, N. (2008). Does family ownership shape performance outcomes?. Journal of Business Research, 61, 609-614. https://doi.org/10.1016/j.jbusres.2007.06.035 
Smith, B. F. y Amoako-Adu, B. (1999). Management succession and financial performance of family controlled firms. Journal of Corporate Finance, 5, 341-368. https://doi. org/10.1016/S0929-1199(99)00010-3

Sraer, D. y Thesmar, D. (2007). Performance and behavior of family firms: Evidence from the French stock market. Journal of the European Economic Association, 5, 709- 751. https://faculty.haas.berkeley.edu/dsraer/sraerthesmar.pdf

Stewart, B. (1991). The Quest for value: A guide for senior managers. New York: Harper business.

Torres, M., Paz, K., y Salazar, F. G. (2006). Métodos de recolección de datos para una investigación. Rev. Electrónica Ingeniería Boletín, (3), 12- 20.

Villalonga, B. y Amit, R. (2006). How do family ownership, control and management affect firm value?. Journal of Financial Economics, (80), 385-417. https://doi.org/10.1016/j. jfineco.2004.12.005

Westhead, P. y Howorth, C. (2006). Ownership and management issues associated with family firm performance and company objectives. Family Business Review, 19, 301316. http://citeseerx.ist.psu.edu/viewdoc/download?doi=10.1.1.889.7136\&rep=rep1\&type $=$ pdf

Yammeesri, J. y Lodh, S. C. (2004). Is family ownership a pain or gain to firm performance. Journal of American Academy of Business, 4(1/2), 263-270. https:/www.researchgate.net/publication/292704853_Is_family_ownership_a_pain_or_gain_to_firm_ performance

Zahra, S. A. (2003). International expansion of US manufacturing family businesses: The effect of ownership and involvement. Journal of Business Venturing, 18(4), 495-512. https://experts.umn.edu/en/publications/international-expansion-of-us-manufacturing-family-businesses-the 